Cahiers de philosophie de l'université de

CAHIERS DE PHILOSOPHIE
DE L L UNIVERSTIE DE CAEN

Caen

47 | 2010

Le phénomène Europe

\title{
L'Europe, les idées et les hommes. Notes sur la lecture derridienne d'une phrase de Husserl
}

Jean-Michel Salanskis

\section{OpenEdition}

1 Journals

Édition électronique

URL : https://journals.openedition.org/cpuc/1133

DOI : $10.4000 /$ cpuc. 1133

ISSN : 2677-6529

Éditeur

Presses universitaires de Caen

Édition imprimée

Date de publication : 15 décembre 2010

Pagination : 61-74

ISBN : 978-2-84133-365-3

ISSN : 1282-6545

Référence électronique

Jean-Michel Salanskis, « L'Europe, les idées et les hommes. Notes sur la lecture derridienne d'une phrase de Husserl », Cahiers de philosophie de l'université de Caen [En ligne], 47 | 2010, mis en ligne le 02 septembre 2020, consulté le 31 janvier 2023. URL : http://journals.openedition.org/cpuc/1133 ; DOI : https://doi.org/10.4000/cpuc. 1133

\section{cc) (1) 8}

Creative Commons - Attribution - Pas d'Utilisation Commerciale 4.0 International - CC BY-NC 4.0

https://creativecommons.org/licenses/by-nc/4.0/ 


\section{L'Europe, les idées et les hommes. Notes sur la lecture derridienne d'une phrase de Husserl}

$\mathrm{M}$ ON BUT, dans cet article, est de discuter et de mettre à l'épreuve l'idée d'une définition idéaliste de l'Europe, telle que celle donnée par Husserl dans sa célèbre conférence de Vienne de 1935.

D’un côté, je voudrais prendre en considération les objections qui ont été formulées à une telle définition, de l'autre, je voudrais prendre au sérieux la difficulté que soulève en l'espèce la conjugaison de l'idéalisme et de la phénoménologie. Mon intention ultime, en effet, est de procurer à la définition idéaliste de l'Europe le soubassement phénoménologique dont on éprouve souvent, et non sans quelque raison, qu'il manque.

Dans une telle entreprise, je dois absolument commencer par examiner la critique de la définition idéaliste de l'Europe par Husserl provenant d'un auteur non extérieur à la phénoménologie, Jacques Derrida.

\section{La charge derridienne}

Dans son ouvrage De l'esprit, publié en 1987 dans la foulée de la première affaire Heidegger en France - qui faisait suite au livre de Victor Farias - Derrida confronte plusieurs discours sur l'Europe émis à l'heure même où le nazisme prenait possession de l'Allemagne. Il rappelle ainsi ce qu'ont pu dire Valéry, Heidegger et Husserl, nous ramenant non seulement à une conjoncture de l'histoire qui n'est plus la nôtre, mais aussi à une ambiance rhétorique perdue.

Dans une note qui court de la page 94 à la page 100 du livre, en particulier, il revient sur une formulation de Husserl, pour établir un étonnant parallèle entre lui et Heidegger. Son point d'appui est le passage suivant, tiré de la conférence de Vienne de 1935 («La crise de l'humanité européenne et la philosophie»), texte célèbre de Husserl, quasi homonyme de son grand ouvrage tardif: 
Nous posons la question: comment se caractérise la figure spirituelle de l'Europe. J'entends l'Europe non pas géographiquement comme sur les cartes, comme s'il était possible de définir le domaine de l'humanité qui vit ici territorialement ensemble, en tant qu'humanité européenne. Au sens spirituel, il est manifeste que les dominions anglais, les États-Unis, etc., appartiennent à l'Europe, mais non pas les Esquimaux ou les Indiens des ménageries foraines, ni les Tziganes qui vagabondent perpétuellement en Europe. Il est manifeste que, sous le titre d'Europe, il s'agit ici de l'unité d'une vie, d'une activité, d'une création spirituelle, avec tous les buts, tous les intérêts, soucis et peines, avec les formations téléologiques, les institutions, les organisations. Dans cet ensemble, les hommes individuels agissent au sein de diverses sociétés de niveau différent, les familles, les tribus, les nations, toutes intérieurement unies spirituellement et, comme je le disais, dans l'unité d'une seule figure spirituelle ${ }^{1}$.

\section{Le commentaire de Derrida se fixe sur une phrase du passage:}

Cette détermination "spirituelle» de l'humanité européenne peut-elle se concilier avec l'exclusion des «Esquimaux, des Indiens des ménageries foraines, ou des Tziganes qui vagabondent perpétuellement en Europe ${ }^{2}$ ?

De là, il en arrive à la mise en balance suivante:

Au titre de l'esprit et de l'Europe, puisque c'est ici notre seul propos, il ne faut pas oublier ce que certaines "victimes» ont écrit et pensé. Et toujours au nom de l'esprit. Heidegger aurait-il souscrit à ce que Husserl a dit des Tziganes? Aurait-il rejeté les «non-aryens» hors de l'Europe, comme le fit celui qui pourtant se savait «non aryen» lui-même, Husserl? Et si la réponse est «non», vraisemblablement «non», est-il sûr que ce soit pour d'autres raisons que celles-là mêmes qui l'éloignaient de l'idéalisme transcendantal? Ce qu'il a fait ou écrit, est-ce pire? Où est le pire? Voilà peut-être la question de l'esprit'.

Au bout du traitement derridien de ce passage husserlien, nous sommes donc conduits à douter qu'il soit facile de discerner, entre ce que Husserl et Heidegger ont "fait ou écrit», le pire. Le dossier produit aurait la force de rétablir l'équilibre, de déstabiliser l'évaluation commune que Derrida vient de rapporter:

1. Cf. E. Husserl, «La crise de l'humanité européenne et la philosophie» [désormais CV], La crise des sciences européennes et la phénoménologie transcendantale, G. Granel (trad. fr.), Paris, Gallimard, 1976, annexe III, p. 352.

2. Cf. J. Derrida, De l'esprit. Heidegger et la question [désormais DE], Paris, Galilée, 1987, p. 95.

3. Ibid., p. 96 . 
On oppose souvent, à juste titre, Husserl et Heidegger, non seulement dans leur pensée mais dans leur histoire politique. Bien qu'il conteste les faits ou les récits, on accuse souvent Heidegger d'avoir participé aux persécutions dont Husserl a été la victime ${ }^{4}$.

Je voudrais, dans ce bref article, revisiter cet «argument derridien » afin d'évaluer sa plausibilité et sa fragilité. Dans l'idée de, peut-être, tirer d'une telle discussion quelques pensées sur l'Europe.

Il faut d'abord réussir à s'étonner de l'opération derridienne. Ce qui est mis en balance, c'est le passage de la conférence de Vienne que nous avons cité plus haut, et, au moins, 1) l'adhésion au parti nazi ; 2) l'acceptation d'une fonction administrative importante à Fribourg donnée par le pouvoir nazi ; 3) l'intervention philosophique du "Discours de Rectorat», dans laquelle Heidegger harmonise certains accents et contenus de sa pensée avec le nouvel élan historique donné à l'Allemagne par Hitler [je m'en tiens ici à des éléments que Derrida connaît et reconnaît]. Comment en arrive-t-on à une telle "rééquilibration" ? C'est ce qui ne me semble pas aller de soi, avant même d'entrer dans le genre philosophique: du strict point de vue du jugement politique. De ce point de vue en effet, on est habitué à distinguer les actes politiques des autres, on est habitué à affecter d'un poids spécifique ce qui se signale soi-même, par des propriétés "pragmatiques", ayant tout à voir avec une convention et un contexte, comme politique: même si c'est pour envisager que des actes différents, s'insérant dans d'autres réseaux qui les identifient d'abord autrement, puissent avoir une "portée politique». La question de ce qui est le pire peut-elle être comprise ici autrement que du point de vue politique? Et la mise en œuvre de ce point de vue n'exige-t-elle pas absolument la distinction des types d'acte indiquée à l'instant? Telle est la raison de ce que j'appelle étonnement: la faute massivement politique de Heidegger ne devrait pas pouvoir être facilement égalisée à la phrase de Husserl.

Essayons pourtant de dégager ce qui justifie pour Derrida son résultat. Procédant de manière régressive à partir de la question finale (« Ce qu’il a fait ou écrit, est-ce pire?»), je commencerai par ce qui la précède immédiatement, et semble lui donner localement le support qui la valide. Comme les choses sont écrites, ce qui nous autorise à douter que le pire soit du côté de Heidegger, c'est le fait que, peut-être, celui-ci n'aurait pas souscrit à ce que Husserl dit des Tziganes. On peut noter à cette occasion qu'il n'est pas clair qu'un doute ait la force de justifier un doute en l'espèce:

4. Ibid. 
pour envisager de douter que Heidegger ait «fait et écrit» pire que Husserl, j'ai le sentiment que, si je partageais l'optique de Derrida, j'aurais besoin d'être sûr qu'il n'aurait pas souscrit à l'énoncé de Husserl sur les Tziganes. À moins de cela, la «différence politique» évoquée à l'instant et qui les sépare continue, me semble-t-il, de prévaloir.

À quoi l'on répondra que les doutes, en l'occurrence, n'en sont pas. Les questions que pose Derrida correspondent à ses thèses. La question "Et si la réponse est "non", vraisemblablement "non", est-il sûr que ce soit pour d'autres raisons que celles-là mêmes qui l'éloignaient de l'idéalisme transcendantal?» l'indique au fond assez clairement: la conditionnelle ne met pas en suspens le fait que la réponse soit «vraisemblablement "non" ", elle l'affirme, et le "est-il sûr que» s'entend comme un opérateur rhétorique introduisant l'interprétation de Heidegger assumée par Derrida. Pour Derrida en effet, la distance prise par Heidegger à l'égard de l'idéalisme transcendantal lui permet de s'abstenir d' "assignations" inspirées par la métaphysique, au rang desquelles il classe celle qu'il trouve chez Husserl.

Cependant, si nous ne partageons pas une telle interprétation, si nous en revenons à l'interrogation nue portant sur ce à quoi Heidegger aurait souscrit, il est beaucoup plus difficile de conclure et de déterminer le vraisemblable que ne paraît le reconnaître Derrida. On peut en effet aborder la question soit à partir du niveau politique, soit par induction à partir de ce que Heidegger a écrit dans la même période. Au niveau politique, c'est bien Heidegger et non Husserl qui a donné son adhésion au parti nazi, dont les intentions à l'égard des Tziganes - au bout du compte exprimées par la pratique systématique du meurtre - ont été ce que l'on sait. Si l'on s'en tient à ce qu'il écrit dans les années en cause, alors nous avons trace, comme Derrida lui-même y insiste et le commente, d'une célébration philosophique par Heidegger de la décision de soi nazie du peuple allemand, prise comme expression de l'aventure vraie de l'Esprit, comme modalité de son affirmation authentique privilégiée: cela pourrait suffire, il me semble, à endosser une description dévalorisante à l'endroit de populations manifestement extérieures à cette décision.

En d'autres termes, Derrida paraît superposer et confondre, ici, la valeur que la philosophie de Heidegger a pour lui - celle d'une suspicion des prédications assignantes piégées par la métaphysique, cette dernière étant elle-même comprise comme la synthèse et l'horizon ultime de toutes les «idéologies» de tous les pouvoirs - avec ce qu'on pourrait appeler la grammaire philosophico-politique de Heidegger à cette époque. Sa prédiction favorable sur Heidegger reflète en l'occurrence une appropriation «française de gauche» de la pensée de Heidegger dont il n'est pas clair 
qu'elle soit juste d'une part, dont il est extrêmement douteux qu'elle soit un outil prédictif adéquat vis-à-vis de la question posée dans le contexte où elle l'est d'autre part.

Mais cette première plongée dans l'analyse derridienne reste encore à la surface des choses. Il y a un choix qui se situe avant l'enchaînement de questions menant au doute que nous venons de soupeser: c'est celui, simplement, de comprendre que Husserl a exclu Indiens, Esquimaux et Tziganes de l'Europe spirituelle.

Ce que dit Husserl, littéralement, est que les Esquimaux, les Indiens des ménageries foraines, et les Tziganes n'appartiennent manifestement pas à l'Europe, entendue en son sens spirituel. Un tel constat de non-appartenance vaut-il comme exclusion?

Ce que Husserl appelle l'Europe spirituelle, nous le lisons dans l'ensemble de la conférence, c'est l'humanité assumant la «tâche infinie» de la connaissance de tout étant au sein de l'universum des étants, assumant le mode de vie de l'autonomie rationnelle qui s'associe à cette tâche. En telle sorte que ne pas appartenir à cette Europe spirituelle signifie simplement ne pas avoir pris sur soi jusqu'à présent cette tâche, ou, de manière un peu plus lâche, habiter un monde culturel qui ne se relie nullement au réseau enchevêtré des institutions et des pratiques par lesquelles et dans lesquelles s'organise collectivement l'assomption de cette tâche. Si j'envisage parfaitement qu'on puisse contester la justesse de l'évaluation de non-appartenance de Husserl au moment où il la commet, je ne vois pas pour autant qu'on puisse la considérer comme une "exclusion» au sens d'un rejet $a$ priori, au sens du refus de la carte de séjour pour les populations concernées au sein de l'Europe spirituelle.

L'Europe spirituelle telle que Husserl la conçoit est clairement une école avant tout, et le texte husserlien me semble assez explicite sur l'adresse universelle de cette école. Par exemple il écrit:

Dans ce processus [celui de l'Europe spirituelle] l'humanité apparaît comme une seule vie pour les hommes et les peuples, liée seulement par une relation spirituelle, avec une quantité de types d'humanité et de cultures, mais dont les courants se mêlent les uns aux autres 5 .

Ou encore, à la fin du passage même qu'a «stigmatisé» Derrida:

Dans cet ensemble [celui de l'Europe spirituelle] les hommes individuels agissent au sein de diverses sociétés de niveau différent, les familles, les

5. Cf. E. Husserl, CV, p. 353. 
tribus, les nations, toutes intérieurement unies spirituellement et, comme je le disais, dans l'unité d'une seule figure spirituelle ${ }^{6}$.

En d'autres termes, la seule interprétation possible du texte de Husserl me semble être qu'il repère une inappétence jusqu'à nouvel ordre pour le télos de l'Europe spirituelle chez ceux qu'il désigne, sans énoncer en aucune manière une inadaptation ou une incompétence essentielle. Qu'il puisse avoir factuellement tort d'une part, qu'on puisse d'autre part lui faire grief de la simple mention de ce "non-rattachement jusqu'à nouvel ordre", au titre qu'il peut être ressenti comme blessant par un lecteur, ou au titre qu'il peut avoir une résonance pragmatique excluante, c'est fort possible, et cela prouve éventuellement que tout rationalisme doit se surveiller luimême avec plus de vigilance et d'exigence que ne l'a fait Husserl. Mais il n'y a pas là pour autant un cas d'ouverture de la philosophie à la malignité politique du même type et du même ordre que ce qui est en discussion chez Heidegger.

D'ailleurs, le surlignement derridien ignore aussi le mouvement essentiel du texte, qui est l'affirmation de la non-géographicité de l'Europe. L'Europe au sens de Husserl, c'est finalement la «tâche infinie» de la «science-philosophie» avec sa retombée sur la production de normes individuelles et communes. Les « exemples» de Husserl - négatifs et positifs - sont essentiellement là pour rappeler que la localisation géographique, en la matière d'une telle Europe, ne saurait être un critère ni une garantie. C'est ce qui rend très étrange le supplément critico-ironique apporté par Derrida dans sa note, lorsqu'il écrit:

Il faudrait donc, pour sauver les dominions anglais, le pouvoir et la culture qu'ils représentent, distinguer par exemple entre les bons et les mauvais Indiens ${ }^{7}$.

Que veut dire Derrida? Est-ce que, pour lui, si les dominions anglais sont rattachés à l'Europe spirituelle, les Indiens d'Inde le sont ipso facto, et du coup sont traités différemment de ceux des «ménageries foraines » ? Ce qui serait à ses yeux une contradiction grotesque? Mais de quel droit appliquerions-nous à la sous-localité du dominion un principe d'homogénéité géographique que justement tout le propos de Husserl vise à contester? Les exemples de Husserl invitent à un discernement qui ne prenne jamais la localité pour preuve d'Europe, pas plus que l'appartenance à un peuple d'ailleurs (faudrait-il que Husserl tranche globalement pour « tout Indien »,

\footnotetext{
6. E. Husserl, CV, p. 352.

7. Cf. J. Derrida, DE, p. 95.
} 
pour qu'il redevînt respectable ?). À vrai dire, Husserl énonce un peu plus loin que les individus d'une nation d'abord repérée comme se rattachant à l'Europe spirituelle peuvent parfaitement ne pas être habités par le télos européen ${ }^{8}$. Il en résulte qu'une description fine devrait prendre en compte une sorte de double gradualité: celle des rattachements personnels, et celle des «institutionnalisations» du télos dans des régions ou cultures particulières. Ce genre de description conduisant à des évaluations qui, en tout cas, ne sont pas directement dictées par le lieu ou par le fait ethnique.

Encore une fois, rappelons que, par ailleurs et au-delà de tout cela, il y a en effet un problème éthique spécifique associé à l'émission même des meilleurs jugements possible en la matière, les plus raffinés et les plus labyrinthisés, problème résultant de leur résonance possible: la non-participation de certains groupes à un télos que l'on valorise, que l'on prend à vrai dire comme le cœur de toute valeur, ne peut pas être "dite-adressée» comme une stabilité factuelle sans risquer au moins de faire tort. Faut-il alors pousser la sensibilité dans une telle matière jusqu'à interdire tout constat sur la "réception" de la tradition rationnelle, ou sur l'extériorité à certains horizons jusqu'à nouvel ordre de ceux qui se trouvent pris dans certaines situations? Je comprends qu'on puisse le penser, mais ce qui me gêne est que, par ailleurs, il ne saurait y avoir école sans reconnaissance qu'une certaine transmission n'a pas encore eu lieu. Dès lors l'ambition que l'humanité dans son ensemble se mette à l'école de certains contenus et certaines méthodes exige que l'on puisse faire ici et là le constat négatif de leur non-transmission: ne pas le faire, serait, précisément, «exclure» les êtres humains concernés en les regardant comme non manquant de ce qui ne cesse de nous manquer à mesure que nous nous mettons à son école.

Pour conclure sur l'intervention derridienne, juste une dernière remarque: une des questions de Derrida (à propos de Heidegger) s'énonce "Aurait-il rejeté les "non-aryens" hors de l'Europe, comme le fit celui qui pourtant se savait "non aryen" lui-même, Husserl?». Elle contient un glissement de plus: celui de traduire le propos de Husserl non seulement comme un acte d'exclusion, mais comme un rejet des non-aryens: ce qui, si mon oreille sémantique n'est pas trop fausse, sous-entend au moins que Husserl se serait livré en l'occurrence à un rejet des non-aryens comme tels. Cette fois, la distance avec ce qui est effectivement dit dans l'orientation où c'est dit atteint son comble, et je ne réussis pas à ressentir ce texte de Derrida autrement que comme une fleur de l’agressivité rusée.

8. Cf. E. Husserl, CV, p. 355. 
C'en est assez pour le retour sur cette incisive note. La raison pour laquelle il m'a paru important de l'interroger, pour comprendre quelles logiques traversaient l'étonnante rééquilibration qu'elle opérait, est simplement son extraordinaire succès. Bien entendu, les admirateurs de la pensée heideggerienne la citent volontiers lorsqu'il s'agit de discuter sur l'ombre portée par l'engagement de Heidegger sur son message. Mais, au-delà, elle est prise en considération comme un jalon de la pensée "critique" par nombre de ceux qui, de par le monde, se rattachent à celle-ci après l'épisode glorieux de la «French Thought». La pensée de cette note derridienne, en effet, est une ressource pour tous ceux qui veulent maintenir une critique de l'«Occident capitaliste» non différenciée dans sa gravité et sa virulence de la condamnation du nazisme. Pour cette critique, il est très important de disqualifier les paroles de l'idéalisme, de montrer qu'elles sont aussi coupables que les pensées de la tragédie et de la vacillation rationnelle: on en déduit que nous avons indéfiniment besoin de ces dernières pour dénoncer le faux-semblant du monde dominant, à quoi qu'elles aient pu conduire et par quoi qu'elles aient pu se laisser séduire.

\section{Validité et force phénoménologique de la définition idéaliste}

D'où la section ultime de cette réflexion. Qu'est-ce que tout cela a à voir avec l'Europe? Husserl présente en résumé l'Europe comme quelque chose de déjà projeté ailleurs, de non complètement établi «chez soi», et qui provient d'une époque perdue d'un pays méditerranéen. L'Europe de Husserl, c'est quelque chose que tout le monde peut s'approprier et personne à la fois. Ou l'intermédiaire entre un passé extrêmement localisé (la Grèce), et un futur visiblement mondial. Quel contraste, à cet égard, avec la vision de Heidegger, pour qui l'Europe «se trouve dans un étau entre la Russie et l'Amérique, qui reviennent au même quant à leur appartenance au monde, ou plutôt à leur caractère de monde et à leur rapport à l'esprit " 9 ! Plutôt que d'être destinalement projetée partout, l'Europe de Heidegger est menacée sur tous ses bords par le «caractère de monde» dominant: peut-il rester un horizon d'Europe à cette Europe acculée, ou se trouve-t-elle vouée à «sortir» du monde sur place, à la faveur d'une relance anti-idéaliste et anti-techniciste? Perspective dont il est douteux qu'elle annonce la moindre multiplicité humaine ou pensante.

9. J. Derrida, DE, p. 93. 
Mais l'enseignement de Husserl, de son côté, veut-il dire que la «vieille Europe» ne mérite aucun amour philosophique ou phénoménologique? Dissout-il par avance l'Europe historique dans une téléologie rationnelle dont le seul niveau adéquat est le monde? C'est à ce sujet que je voudrais pour finir essayer quelques réflexions.

Pour qui écrit aujourd'hui en Europe, dans l'un de ses vieux pays, et cependant que depuis plus d'un demi-siècle se poursuit un étrange effort baptisé «construction européenne », la question cruciale qui se pose, on le sait, est celle de la promotion de l'Europe au rang d'objet collectif de pleine stature, c'est-à-dire d'objet à la fois reconnu et "co-vécu». On est dans l'attente du moment où un «sentiment européen » aurait vu le jour, ce qui devrait aller de pair, imagine-t-on, avec l'émergence d'une idée européenne. Étant bien entendu qu'il faudrait que l'Europe ainsi véritablement fondée ne soit pas l'Europe illimitée, mais qu'elle soit l'Europe géographique, celle que nous connaissons déjà dans un repérage conventionnel et confortable. Faut-il, pour fonder et défendre une telle Europe, tourner le dos à l'Europe de Husserl, parce que celle-ci ne saurait s'abstenir d'intégrer la Corée, le Brésil et le Nigeria? Faut-il considérer que l'origine grecque désignée par Husserl ne se laisse en aucune manière approprier par le «continent» (en vérité sous-continent) européen tel que nous le fréquentons et cherchons à le faire exister politiquement? À l'inverse de Jacques Derrida, la difficulté que nous voulons explorer à propos de l'Europe de Husserl est celle de son inclusivité absolue.

Il me semble qu'on peut justifier l'étape symbolique de l'Europe historique de deux manières: d'une part, en termes de mémoire, d'autre part, en termes de personnes. Et ce, sans récuser la notion husserlienne de l'Europe spirituelle, héritière de la tâche infinie de la science et de la vie normative accompagnant la science. De l'une ou l'autre de ces manières s'esquisse, peut-être, une attestation phénoménologique de l'Europe, qui ne contredise pas sa construction idéaliste par Husserl.

En termes de mémoire, d'abord. L'idée est contenue dans la locution «étape symbolique» osée à l'instant: l'Europe familière, de fait, a été le cadre de la progression de la figure et de l'entreprise de l'Europe spirituelle. Sur son territoire, de fait, des mutations décisives ont été engagées: celle de l'encyclopédisme à la Renaissance (apportant une vision du tout du savoir dont l'importance est extrême), celle de la révolution scientifique (qu'il faille la lire dans la percée de Galilée, dans le travail des expérimentateurs anglais de la fin du XVIII ${ }^{\mathrm{e}}$ siècle ou dans celui des mathématiciens français du début du XIX ${ }^{\mathrm{e}}$ siècle), aussi bien que celle de la révolution démocratique (qu'il faille l'associer à la Révolution anglaise partielle du $\mathrm{XVII}^{\mathrm{e}}$ siècle ou à la Révolution française, et même si, sur ce point, la 
«nouvelle Europe» au-delà de l'Atlantique est dans le jeu d'emblée). À quoi j'ajouterai l'expérience du mal que porte le lieu européen, celui des guerres de religions, celui du gaspillage meurtrier des vies à grande échelle en 1914-1918, ou celui du crime mondial a priori que fut l'extermination au cœur de la chevauchée guerrière nazie de 1939-1945. Cette expérience, et sur ce point je comprends Derrida, est liée à la figure de l'Europe spirituelle, elle est l'expérience de ce que son grand mouvement ne sait pas empêcher par lui-même. Elle doit donc désormais hanter l'exercice de la «normativité accompagnant le développement infini de la science » comme l'indication d'une sorte de condition limite de validité : si le monde où vit et prospère la «tâche infinie» de Husserl laisse «revenir » la monstruosité du crime, alors cette tâche y perd complètement ses droits et son sens.

L'Europe familière fut le théâtre d'un segment considérable de l'aventure de «l'Europe spirituelle», en telle sorte que cette dernière garde une dette à son endroit d'une part, en telle sorte que, d'autre part, la constitution d'une Europe politique offrirait une chance herméneutique exceptionnelle à l'Europe spirituelle: dans une telle Europe, la possibilité de comprendre comment se sont noués tous les fils de l'affaire spirituelle au cours du segment historique européen serait ouverte mieux et plus profondément que dans l'état actuel des choses. Du point de vue de la poursuite de la tâche infinie, cette Europe n'aurait aucun privilège, mais du point de vue de la compréhension de la dette de la tâche infinie à l'égard du moment européen, elle apporterait des ressources extraordinaires. Par conséquent, si nous comprenons que les choses de l'esprit passent éminemment par la dette, qu'il n'y a pas d'intelligence oublieuse, que la manière dont les progrès ont été conquis et acquis compte pour l'éternité, alors nous voyons une signification éminente pour l'Europe politique, économique et culturelle: sa réunification permettrait de rendre manifeste dans son histoire, en en faisant le partage de millions d'hommes, l'aventure enchevêtrée d'une longue et essentielle phase de l'Europe spirituelle.

Mais cet enchevêtrement lui-même, nous pouvons le comprendre de manière concrète en prenant en considération le niveau de la personne, ainsi que nous l'annoncions plus haut. Ce qui s'est passé en Europe, ce que fut l'Europe en tant que lieu de l'Europe spirituelle, est lié à des trajectoires de personnes. Bien avant l'Europe politique, institutionnelle ou économique, les itinéraires de personnes vivant déjà sous condition européenne firent briller un possible transnational.

On peut penser ici aux voyages d'Érasme, donnant encore leur nom aux mobilités étudiantes de nos jours. À ceux de Descartes ou de Leibniz, à Maupertuis prenant la direction de la très récente Académie de Berlin. À la 
vie de combats et d'exils de grands idéalistes nationaux comme Kosciuszko. Ou même tout près de nous au jeune Russell se rendant à Berlin où il étudie les travaux du mathématicien Felix Klein, ou venant au congrès de philosophie de Paris de 1900, invité par Couturat. Ou encore, à Levinas venant de Lituanie à Paris en passant par Strasbourg (et, un peu, Fribourg). Mais combien de noms faudrait-il citer, dans les arts, les sciences, la littérature, la médecine, le droit, la politique, etc. ? L'Europe spirituelle à laquelle pense Husserl, elle a trouvé aussi sa consistance (relative) dans les cheminements de ceux qui se déplaçaient d'un territoire de l'Europe historique à un autre au nom de la poursuite d'une «condition» idéale, pour rejoindre des livres, des idées, d'autres personnes engagées dans la mise en œuvre d'un fragment de la «tâche infinie». L'Europe historique reste marquée par les mobilités qui l'ont sillonnée au nom d'autre chose que sa base géographique. Et en même temps, cette géographie, comme toute géographie, a été aussi, au long des siècles, facteur de mélanges, d'exils, d'immigrations : la contiguité des pays de l'Europe historique les a également «brassés " pour ainsi dire en amont de la "tâche infinie", précipitant les mariages, les voyages, les déplacements de travailleurs. L'idée de l'Europe veut le renforcement mutuel de ces expériences qui ne sont pas seulement incommensurables: avoir affaire avec ses voisins n'est pas étranger à la volonté de la tâche infinie, même si cette dernière n'est pas donnée comme telle dans toute connexion «économique» au sens large.

L'Europe, si elle se fait "jusqu'au bout», reconnaîtra toutes ces trajectoires comme une part de sa chair, comme ce par quoi passait et se concrétisait le rattachement à la tâche infinie. La partie de l'Europe spirituelle appelée à continuer la "tâche infinie » dans les limites de l'Europe historique se laissera identifier par la garde et l'interprétation de la mémoire des épisodes de l'Europe spirituelle liés à son territoire, et par l'assomption - comme son «corps spirituel» en quelque sorte - des itinéraires faiseurs d'Europe des personnes de son histoire. L'espoir que l'on peut associer à la venue au jour d'une telle Europe est que sa contribution à la «tâche infinie» affiche une spécificité résultant de cette garde et de cette assomption, spécificité profitable au débat absolument universel de l'Europe spirituelle.

Reste à envisager quelque chose comme une attestation phénoménologique du nous de l'Europe. Sartre, on le sait, distingue à cet égard deux niveaux ou deux figures, celles du nous sujet et du nous objet. Suivant le nous sujet, je m'expérimente comme jalon ou relais dans un réseau anthropologique où s'insèrent des outils, des signes et des hommes (j'utilise le couloir mécanique de la station Châtelet, par exemple) : c'est une manière de me découvrir dans le vécu membre d'un nous. La figure du nous objet 
correspond au cas où un tiers me thématise avec d'autres, au cas où je me trouve lié à d'autres dans la commune prise intentionnelle d'une visée qui nous objective tous de conserve.

Canetti, dans Masse et puissance, propose une théorie de l'appartenance nationale: les adeptes d'une nation sont ceux qui, ensemble, contemplent une même image, sorte de schème effectuant la nation pour tous. Ainsi, les Britanniques contemplent ensemble les mers qui environnent leurs îles, les Allemands contemplent leurs forêts, les Suisses leurs montagnes (tout alpiniste est un Suisse d'honneur) et les Français contemplent leur Révolution, selon lui. Ce repérage phénoménologique me semble intermédiaire entre le nous sujet et le nous objet: il renvoie à un éprouver en première personne, mais celui-ci ne prend pas en vue une structure articulant les compatriotes; et, en fin de compte, on peut se demander si chaque adepte de la nation n'est pas regardé par l'image et subsumé par elle plutôt qu'il ne la contemple.

Il me semble que, dans le cas de l'Europe, nous pouvons alléguer des expériences qui visent l'Europe «du dehors», comme celle des jeunes Américains qui font un voyage en Europe, avant de commencer leurs études universitaires par exemple. Plus généralement, plusieurs ensembles nationaux de la planète sont "post-européens » : il leur est intrinsèque de viser l'Europe comme ce après quoi et de quoi ils viennent (le Canada, l'Amérique du Sud, l'Australie...). Israël est à la fois post-Europe, par une dimension essentielle, et post-post-Europe, dans la mesure où s'y installent aussi des gens qui viennent de pays post-européens. Dans son principe, Israël est à vrai dire post-monde, mais cela n'est pas notre sujet.

Revenant à Sartre, on serait tenté de dire que ces expériences des ressortissants post-européens fondent tout au plus un nous objet: mais le nous objet n'est pas vraiment phénoménologique, il ne semble pas avoir la faculté de présenter originairement la signification du nous en cause. Notre exemple nous conduirait pourtant, je crois, à relativiser cette distinction. Le regard sur l'Europe des citoyens américains nous est mis en scène dans mille histoires rencontrées dans des livres, des films et des séries télévisées. Par le biais de l'identification que suscitent toutes ces narrations, nous sommes amenés à voir l'Europe à travers le regard ainsi mis en scène. Le nous objet en question se subjective de la sorte, et devient capable de participer à la constitution ou à l'attestation.

\section{Mot final: l'Europe, la science, la philosophie}

Si la phénoménologie peut être convoquée pour essayer de déterminer s'il est possible de combler un certain déficit phénoménologique concernant l'Europe aujourd'hui, on peut aussi poser une question plus proche de celle 
que Husserl se posait dans la conférence de Vienne: on peut se demander si la phénoménologie a plus son mot à dire que d'autres composantes de la philosophie, pour appeler et pour mettre en œuvre une Europe idéaliste donnant sens à l'épisode géographique et historique désigné par le nom Europe. Husserl le pensait sans doute, parce que, pour lui, la phénoménologie était la philosophie elle-même dans son essence, et, comme telle, de jure l'animatrice et l'inspiratrice de la formulation et la mise en œuvre de la tâche infinie. Mais devons-nous le penser aujourd'hui de la même manière?

L'auteur de ce petit article se ressent comme phénoménologue, comme guidé dans son type de travail par la tradition de la phénoménologie, mais pourtant, il aspire par exemple à la venue au jour d'une authentique «philosophie analytique européenne», qui intégrerait à l'exercice méthodique de la discussion analytique la mémoire et les personnes. Une couleur européenne peut d'ailleurs affecter, de la même manière, les neurosciences et la littérature. La phénoménologie a seulement comme privilège, en l'occurrence, d'être une démarche d'ores et déjà reprise de façon majeure et importante dans plusieurs pays de la vieille Europe (sans discussion possible, en Allemagne, République tchèque et en France), en telle sorte que, il est vrai, elle possède une aura «continentale» immédiate. Mais voulant l'Europe, nous ne souhaitons pas qu'on en reste là.

Jean-Michel SALANSKIS 